\title{
GJ 1214b and the prospects for liquid water on super Earths
}

\author{
Leslie A. Rogers ${ }^{1}$ and Sara Seager ${ }^{2}$ \\ ${ }^{1}$ Department of Physics, Massachusetts Institute of Technology \\ 37-602, 77 Massachusetts Ave., Cambridge, MA 02139, USA \\ email: larogers@mit.edu \\ ${ }^{2}$ Department of Earth, Atmospheric, and Planetary Sciences, Department of Physics \\ Massachusetts Institute of Technology \\ 54-1626, 77 Massachusetts Ave., Cambridge, MA 02139, USA \\ email: seager@mit.edu
}

\begin{abstract}
GJ 1214b is one of the first discovered transiting planets having mass $\left(6.55 M_{\oplus}\right)$ and radius $\left(2.678 R_{\oplus}\right)$ smaller than Neptune. To account for its low average density $\left(1870 \mathrm{~kg} \mathrm{~m}^{-3}\right)$, GJ 1214b must have a significant gas component. We use interior structure models to constrain GJ 1214b's gas envelope mass, and to explore the conditions needed to achieve within the planet pressures and temperatures conducive to liquid water. We consider three possible origins for the gas layer: direct accretion of gas from the protoplanetary nebula, sublimation of ices, and outgassing from rocky material. Despite having an equilibrium temperature below $647 \mathrm{~K}$ (the critical temperature of water) GJ $1214 \mathrm{~b}$ does not have liquid water under most conditions we consider. Even if the outer envelope is predominantly sublimated water ice, in our model a low intrinsic planet luminosity (less than $2 \mathrm{TW}$ ) is needed for the water envelope to pass through the liquid phase; at higher interior luminosities the outer envelope transitions from a vapor to a super-fluid then to a plasma at successively greater depths.
\end{abstract}

Keywords. Stars: individual (GJ1214), planetary systems

\section{Introduction}

The MEarth transiting planet, GJ 1214b (Charbonneau et al. 2009), is exciting for many reasons. Firstly, it lies in a mass and density regime for which there are no solar system analogs; with $M_{p}=6.55 \pm 0.98 M_{\oplus}$ and $R_{p}=2.678 \pm 0.13 R_{\oplus}$ GJ $1214 \mathrm{~b}$ is smaller than the ice giants Neptune and Uranus, while larger than the terrestrial Earth, Venus, and Mars. Secondly, GJ 1214b has a low enough density $\left(\rho_{p}=1870 \pm 400 \mathrm{~kg} \mathrm{~m}^{-3}\right)$ that it cannot be composed of rocky and iron material alone, and almost certainly contains a gas component. Finally, orbiting with a $1.5803952 \pm 0.0000137$ day period around an $L_{*}=0.00328 \pm 0.00045 L_{\odot} \mathrm{M}$ dwarf, GJ $1214 \mathrm{~b}$ has an equilibrium temperature below $647 \mathrm{~K}$, the critical point of water $\left(T_{e q}=555 \mathrm{~K}\right.$ for a Bond albedo of 0$)$.

We use planet interior structure models to constrain the bulk composition of GJ 1214b. With our current knowledge of GJ1214b (its mass, radius and orbit) we can not pinpoint GJ 1214b's unique true interior composition (see, e.g. Valencia et al. 2007; Adams et al. 2008), but we can quantify the range of possibilities that are consistent with the transit and radial velocity observations. In this proceedings we focus on bounding the mass of GJ 1214b's gas envelope and on studying GJ 1214b's prospects for harboring liquid water. We examine three possible sources for the GJ 1214b gas layer (direct accretion of gas from the protoplanetary nebula, sublimation of ices, and outgassing from rocky material) and consider end-member scenarios in which only one gas-layer source was important in forming the current gas layer on GJ 1214b. 


\section{Model}

We model GJ 1214b as a spherically symmetric differentiated planet in hydrostatic equilibrium. We consider up to four layers within the planet: an iron core, silicate mantle, ice layer, and gas envelope. The gas envelope is assumed to be in radiative-convective equilibrium, with a thin outer radiative zone surrounding a convective zone at greater depths. Within the outer radiative zone we adopt the temperature profile from Equation (45) of Hansen (2008), an analytic "two-stream" solution to the gray equations of radiative transfer for a plane-parallel irradiated atmosphere. The onset of convective instabilities $\left(0<(\partial \rho / \partial s)_{P} d s / d m\right)$ determines depth of the transition to the convective layer of the gas envelope. In the convective regime, we adopt an adiabatic temperature profile.We neglect thermal effects within the core, mantle and ice layer since at the high pressures found in these interior layers, thermal corrections have only a small effect on the mass-density (Seager et al. 2007). A more detailed description of our model (including the equations of state employed) can be found in Rogers \& Seager (2010a) and Rogers \& Seager (2010b).

\section{Results}

\subsection{Direct Accretion Scenario}

We first consider the possibility that GJ 1214b's gas layer originated from direct accretion from the protoplanetary disk and comprises a roughly solar mixture of $\mathrm{H} / \mathrm{He}$. In this case, we take a Neptune-inspired template with four chemically distinct layers in the planet interior: an iron core, silicate mantle, water-ice layer, and $\mathrm{H} / \mathrm{He}$ envelope with $Y=0.28$. Figure 1 shows how the planet mass may be distributed among the four layers and still be consistent with GJ 1214b's measured mass and radius.

In this scenario, GJ $1214 \mathrm{~b}$ requires between $10^{-4}$ and $6.8 \% \mathrm{H} / \mathrm{He}$ by mass to account for the measured planet mass and radius within $1 \sigma$. This range of possible gas layer masses not only includes observational uncertainties, but also uncertainties in the planet's atmospheric pressure-temperature (PT) profile and the iron:silicate:ice mass ratio of the inner three layers. In proportion to the planet mass, the $\mathrm{H} / \mathrm{He}$ envelope on GJ1214b would be more massive than Venus gas layer $\left(\sim 10^{-4} M_{p}\right)$ but less massive than Neptunes $\left(0.05 M_{p}\right.$ to $\left.0.15 M_{p}\right)$.

\subsection{Sublimation of Ices Scenario}

We next explore the scenario in which a layer of vapors from sublimated ices accounts for GJ 1214b's transit radius. This could be the case if GJ $1214 \mathrm{~b}$ did not manage to accrete or retain any $\mathrm{H} / \mathrm{He}$ from the protoplanetary disk, yet still formed from ice-rich material beyond the snow line. For this scenario, we model the planet interior with an iron core, silicate mantle, and water envelope and show in Figure 2 how GJ 1214b's mass may be distributed between these three layers. A sublimated vapor dominated envelope on GJ $1214 \mathrm{~b}$ is possible only if water accounts for a large fraction of planet mass. To account for the observed planet mass and radius within their 1, 2, and $3 \sigma$ observational uncertainties, at least $47 \%, 24 \%$, and $6 \%$ water by mass are required, respectively.

GJ 1214b does not contain liquid water in any of our model interiors displayed in Figure 2. Instead, the putative GJ $1214 \mathrm{~b}$ water envelopes begin in the vapor phase at low pressures, then continuously transition to a super-fluid at $P=22.1 \mathrm{MPa}$ (the critical pressure of water), before eventually becoming an electronically conductive dense fluid plasma at greater depths. To obtain liquid water in our model interior, we must decrease GJ 1214b's assumed intrinsic energy flux below $5 \times 10^{-4} \mathrm{~W} \mathrm{~m}^{-2}$. For comparison, Earth's 


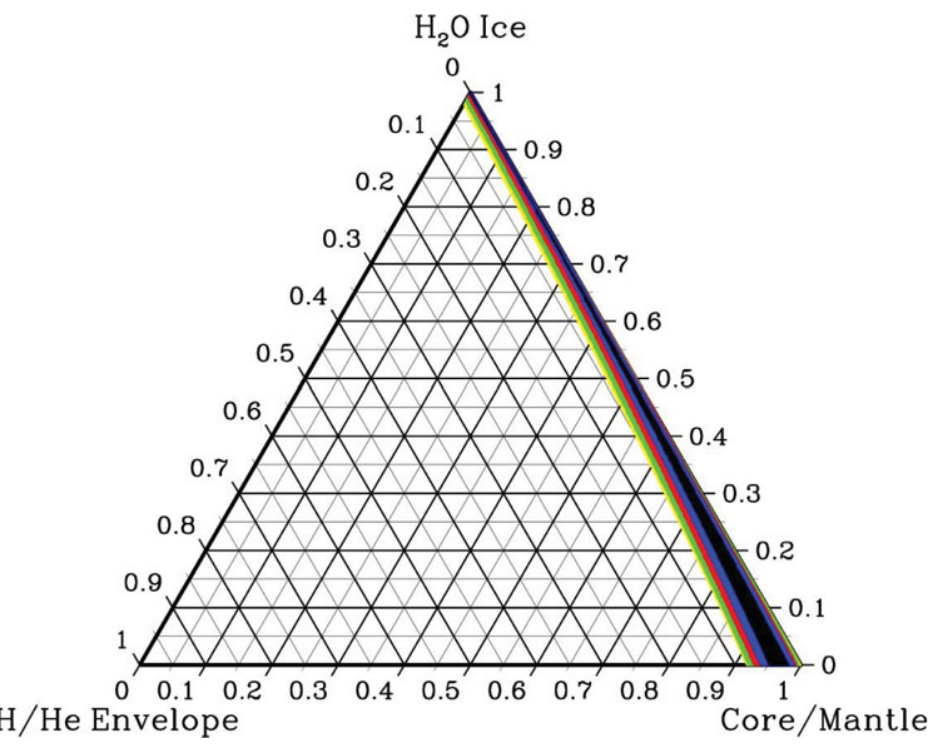

Figure 1. Ternary diagram for the scenario where GJ 1214b's gas layer formed through direct accretion of $\mathrm{H} / \mathrm{He}$. The relative contributions of the iron core, $\mathrm{Mg}_{0.9} \mathrm{Fe}_{0.1} \mathrm{SiO}_{3}$ silicate mantle, $\mathrm{H}_{2} \mathrm{O}$ ices, and $\mathrm{H} / \mathrm{He}$ envelope to the mass of the planet are plotted. The core and mantle are combined together on a single axis, with the perpendicular distance from the lower right vertex determined by the fraction of the planet's mass in the two innermost planet layers. The black shaded region denotes the interior compositions that are consistent with the nominal planet mass and radius $\left(M_{p}=6.55 M_{\oplus}, R_{p}=2.678 R_{\oplus}\right)$ for our fiducial atmospheric PT profile. The $\mathrm{H} / \mathrm{He}$ mass fraction has a spread in this case due to the range of possible core-to-mantle mass ratios. The span of plausible interior compositions widens to the darkest grey (blue online) shaded area when we consider the uncertainties on the planet's albedo and intrinsic luminosity. Successively lighter shades of grey (colored red, green, and yellow online) denote compositions that are consistent with $M_{p}$ and $R_{p}$ to within 1,2 , and $3 \sigma$ of their observational uncertainties, respectively, when uncertainties in the atmospheric parameters are also included. For an explanation of how to read ternary diagrams, see e.g., Valencia et al. (2007).

internal heat flux is $0.087 \mathrm{~W} \mathrm{~m}^{-2}$ (Turcotte \& Schubert 2002). We therefore predict that, in this scenario, GJ 1214b would need a cold interior in order to harbor liquid water.

\subsection{Outgassing Scenario}

We now explore the possibility that GJ 1214b is a rocky planet with an outgassed atmosphere contributing to its transit radius. In this scenario GJ 1214b formed from rocky planetesimals (without retaining significant amounts of primordial gas or icy material) that when heated during planet formation released volatiles to form the planet's gas envelope.

We find that if GJ 1214b is a rocky super Earth with an outgassed atmosphere, its gas layer would need to be Hydrogen rich. In principle, outgassing can produce a wide range of gas layer compositions depending on the composition of the primordial rocky material (Elkins-Tanton \& Seager 2008; Schaefer \& Fegley 2009). However, there is also a limit on how much gas rocky material can outgas, determined by its initial volatile content and oxidation state. In order for the outgassing scenario to be feasible GJ 1214b's gas envelope must have both a mass low enough to be produced by outgassing and a volume large enough to account for the transit radius. $\mathrm{H}_{2}$ is the only species that both is light enough and can plausibly be outgassed in sufficient quantities (e.g. by ordinary H, L, LL and high iron enstatite EH chondritic material) to satisfy this criterion. 


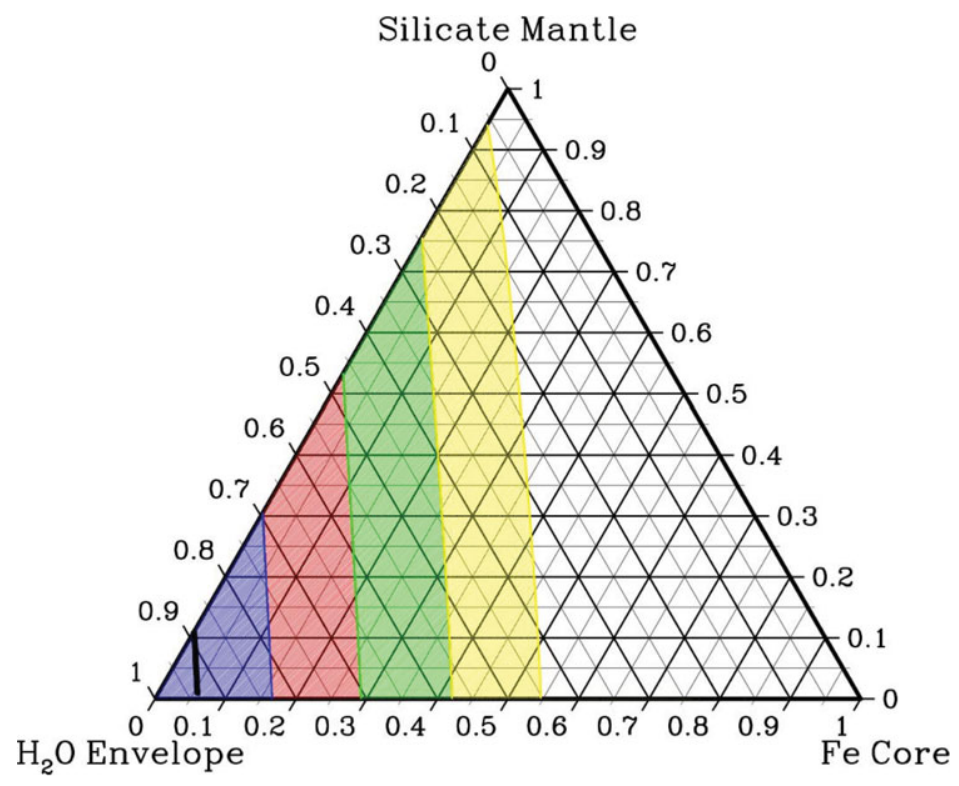

Figure 2. Ternary diagram for the scenario in which GJ 1214b's envelope is dominated by vapor from sublimated water ice. The fractions of the planet's mass in the Fe core, the $\mathrm{Mg}_{0.9} \mathrm{Fe}_{0.1} \mathrm{SiO}_{3}$ silicate mantle, and the water envelope are plotted on the three axes. The solid black curve represents the locus of interior compositions that are consistent with the nominal measured planetary mass and radius for our fiducial PT profile. The colors in this figure have the same designations as in Figure 1.

\section{Summary}

We consider three scenarios for the origin of GJ 1214b's gas layer: direct accretion of nebular gas, sublimation of ices, and outgassing. Based on the measured planet mass and radius alone, none of the scenarios can be ruled out. Future measurements of GJ 1214b's transmission spectrum may help to distinguish between the vapor dominated and hydrogen-dominated cases (Miller-Ricci et al. 2009). We also find that, under most conditions we consider, GJ 1214b would not have liquid water. Its PT profile is simply too hot to go through the liquid water phase.

\section{References}

Adams, E. R., Seager, S., \& Elkins-Tanton, L. 2008, ApJ, 673, 1160

Charbonneau, D., Berta, Z. K., Irwin, J., Burke, C. J., Nutzman, P., Buchhave, L. A., Lovis, C., Bonfils, X., Latham, D. W., Udry, S., Murray-Clay, R. A., Holman, M. J., Falco, E. E., Winn, J. N., Queloz, D., Pepe, F., Mayor, M., Delfosse, X., \& Forveille, T. 2009, Nature, 462,891

Elkins-Tanton, L. T. \& Seager, S. 2008, ApJ, 685, 1237

Hansen, B. M. S. 2008, ApJS, 179, 484

Miller-Ricci, E., Seager, S., \& Sasselov, D. 2009, ApJ, 690, 1056

Rogers, L. A. \& Seager, S. 2010a, ApJ, 712, 974

- 2010b, ApJ, 716, 1208

Schaefer, L. \& Fegley, Jr., B. 2009, ApJ, 703, L113

Seager, S., Kuchner, M., Hier-Majumder, C. A., \& Militzer, B. 2007, ApJ, 669, 1279

Turcotte, D. L. \& Schubert, G. 2002, Geodynamics (Cambridge, UK: Cambridge University Press)

Valencia, D., Sasselov, D. D., \& O'Connell, R. J. 2007, ApJ, 665, 1413 